\title{
Effect of convection modeling on the non-adiabatic seismic observables of $\delta$ Scuti stars
}

\author{
Josefina Montalbán ${ }^{1}$ and Marc-Antoine Dupret ${ }^{2}$ \\ ${ }^{1}$ Institut d'Astrophysique et Géophysique, Université de Liège, 4000- Liége, Belgium \\ email: j.montalban@ulg.ac.be \\ ${ }^{2}$ Observatoire de Paris, LESIA, CNRS UMR 8109, 92195 Meudon France \\ email:ma.dupret@obspm.fr
}

\begin{abstract}
Pulsation frequencies in $\delta$ Scuti stars do not fall in the asymptotic domain, therefore, multi-band photometric methods are currently used to identify the pulsation modes. Theoretical photometric amplitude ratios and phase differences between photometric bands depend, however, on the treatment of convection in surface stellar layers. In this poster we present the results of applying the non-adiabatic analysis by Dupret et al. (2003) to $\delta$ Scuti stellar models computed by using the FST (Canuto et al. 1996, CGM) treatment of convection in the interior and in the atmosphere. We determine the amplitude ratios and phase difference for several bands in the Strömgren photometric system, and we compare the results obtained with the FST treatment and with the classical Mixing-length theory. We show that the differences in the external thermal structure are clearly reflected in the photometric phase differences.
\end{abstract}

Keywords. Convection, stars: oscillations, stars: variables: $\delta$ Scuti

\section{Equilibrium models}

The stellar models were computed by using the evolution code ATON3.0 (D'Antona et al. 2005). All the results presented here corresponds to $1.8 M_{\odot}$ models. Convection is treated by using the classical MLT (Böhm-Vitense, 1958) with the scale length parameter $\alpha=0.1,0.3, \mathbf{0 . 5}, 0.7$ and 1.75, and also by using the FST (Canuto et al. 1996) formalism with the parameter $\beta=0.09$. The surface boundary conditions and the structure for the most external layers are provided by the NEMO atmosphere models (Heiter et al. 2002) computed with both MLT- $\alpha=0.5$ and FST- $\beta=0.09$.

For a given stellar mass and chemical composition, the HR diagram location of a typical $\delta$ Scuti is non affected by the treatment of the non-adiabatic convection layers. All the main sequence (MS) models considered here occupy the same position in the theoretical HRD. Furthermore, the differences between FST and MLT- $\alpha=0.5$ have no observable effects on the stellar spectrum (Heiter et al. 2002). Nevertheless, the temperature gradients and the energy transported by convection are indeed affected by the convection treatment, and we show in this paper that the effects of the different thermal structure on the non-adiabatic observables are not negligible.

\section{Non-adiabatic analysis}

The stellar structure of the equilibrium model is extended by matching the atmosphere structure (from the optical depth $\tau=\tau_{\mathrm{ph}}$ up to $\tau=10^{-4}$ ) obtained by interpolation in the corresponding MLT or FST grids of atmosphere models. We take $\tau_{\mathrm{ph}}=10$ except when $\alpha_{\mathrm{MLT}} \neq 0.5$, in that case $\tau_{\mathrm{ph}}=1$ to be sure that we are in the radiative atmosphere. 

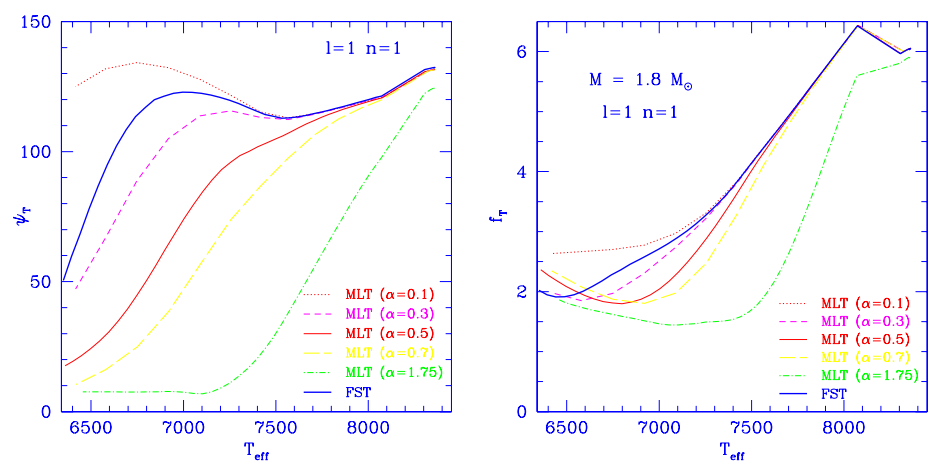

Figure 1. The non-adiabatic quantities $\psi_{\mathrm{T}}$ (left panel) and $f_{\mathrm{T}}$ (right panel) for the mode $\ell=1, n=1$ as a function of the effective temperature for the MS evolution of $1.8 \mathrm{M}_{\odot}$ models computed with different convection treatments.
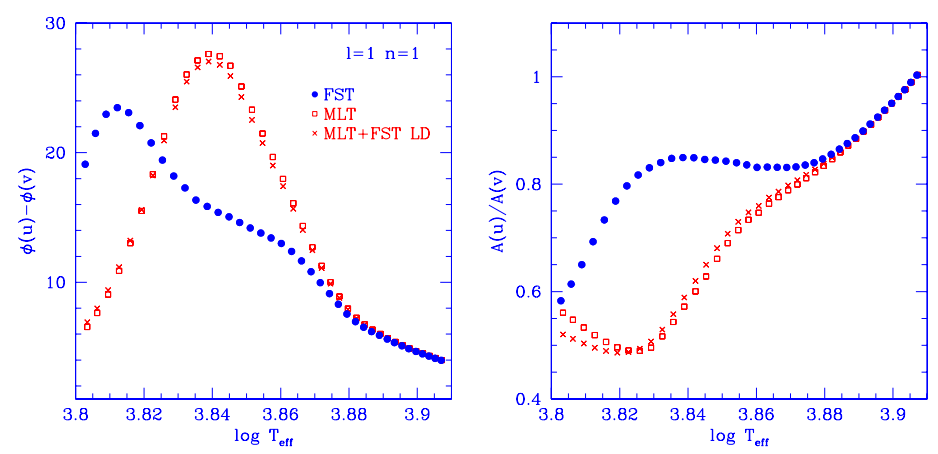

Figure 2. Photometric phase difference (left panel) and amplitude ratio (right panel) for the $u$ and $v$ pass-bands for the same mode and models than in Fig. 1. Solid circles correspond to complete FST models with the color transformation provided by FST atmospheres. Open squares refers to complete MLT- $\alpha=0.5$ models with color transformation given by MLT- $\alpha=0.5$ atmospheres; and crosses correspond to complete MLT- $\alpha=0.5$ and the color transformation given by FST atmosphere models.

The non-adiabatic analysis of these extended models is done by using the code MAD (Dupret et al. 2003) with the "frozen convection" approximation. This analysis provides the quantities $f_{\mathrm{T}}$ (the local relative variation of effective temperature due to pulsation) and $\psi_{\mathrm{T}}$ (the phase-lag between the local variation of effective temperature and the radial displacement). Fig. 1 shows the sensitivity of $f_{\mathrm{T}}$ and $\psi_{\mathrm{T}}$ to the convection treatment for models along the MS evolution of a $1.8 M_{\odot}$ star. The non-adiabatic behavior of the FST models is not equivalent to the MLT one with only an $\alpha$ parameter value. Neither through the star nor along the MS evolution. At high $T_{\text {eff }}$, FST is close to MLT- $\alpha=0.1$ and as $T_{\text {eff }}$ decreases, FST goes closer to $\alpha=0.3$. This change of behavior occurs at $\sim 6800 \mathrm{~K}$, the $T_{\text {eff }}$ at which FST convection regions suddenly become deeper. It is evident that $\psi_{\mathrm{T}}$ is much more sensitive to the convection treatment than $f_{\mathrm{T}}$, and that the differences are larger for colder models than for the hotter ones.

The theoretical photometric observables such as the amplitude ratio and phase difference between the magnitude variations in different photometric bands can be derived from $f_{\mathrm{T}}$ and $\psi_{\mathrm{T}}$, and from the dependence of the monochromatic fluxes and limbdarkening coefficients (LDC) on the effective temperature and gravity. These latest quantities, corresponding to the Strömgren photometric system, have been derived by Barban 


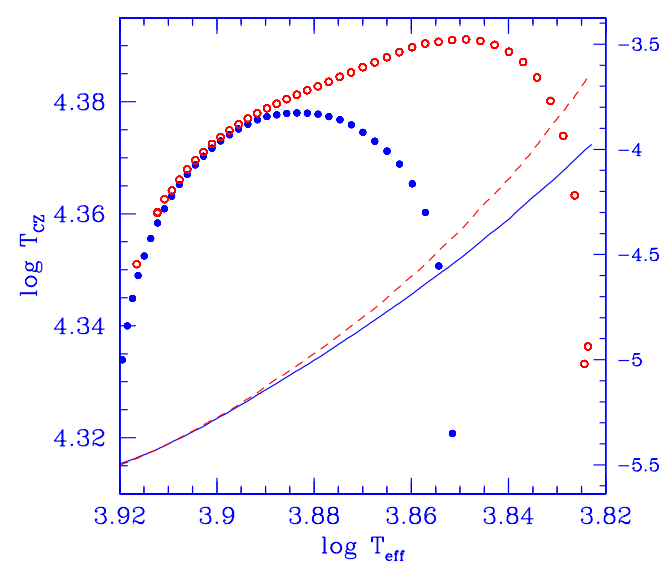

Figure 3. Temperature at the bottom of the convective zone for $1.8 M_{\odot}$ models along the MS evolution: Solid line correspond to FST models, and dashed line to MLT- $\alpha=0.5$ ones. Points represent the value of the imaginary part of the $\ell=0, n=5$ mode frequency. Full circles correspond to FST models and empty symbols to MLT models.

et al. (2003) for the NEMO atmosphere models. In Fig. 2 we show the phase difference (left panel) between the $u$ and $v$ pass-bands, and the amplitude ratio (right panel). We also translate the MLT non-adiabatic quantities to the observational plane by using the LDC corresponding to FST atmospheres (cross symbols), and we see that for the mode $\ell=1, n=1, \phi(u)-\phi(v)$ as well as $A(u) / A(v)$ are mainly sensitive to the thermal structure of the models. Phase differences between two photometric bands are mainly sensitive to the temperature gradient in the hydrogen convection zone. The amplitude ratios, on the other hand, may depend on both, the atmosphere models (through limb-darkening functions) and on the non-adiabatic quantities $f_{\mathrm{T}}$ and $\psi_{\mathrm{T}}$.

Finally, the convection treatment has also a slight effect on the red-edge of the $\delta$ Scuti instability strip. Fig. 3 shows the behavior of $\log \sigma_{\mathrm{I}}$ (with $\sigma_{\mathrm{I}}$ being the imaginary part of the frequency) along the MS evolution of $1.8 M_{\odot}$ star. Log $\sigma_{\mathrm{I}}$ for FST models (full circles) decreases to zero at higher effective temperatures than for MLT- $\alpha=0.5$.

\section{Acknowledgements}

J.M acknowledges financial support from the Prodex 8 COROT (C90199), FNRS and IAU GA grant number 12259 .

\section{References}

Barban, C., Goupil, M.J., Van’t Veer-Menneret, C., Garrido, R., Kupka, F. \& Heiter, U. 2003, A\&A 405, 1095

Böhm-Vitense, E. 1958, Zeitschrift fur Astrophysics 46, 106

Canuto, V., Goldman, I. \& Mazzitelli, I. 1996, ApJ 473, 550

D’Antona, F., Cardini, D., Di Mauro, M.P., Maceroni, C., Mazzitelli, I. \& Montalbán, J. 2005, MNRAS 363, 847

Dupret, M.A., De Ridder, J., De Cat, P., Aerts, C., Scuflaire, R., Noels, A. \& Thoul, A. 2003, $A \mathscr{E} A$ A 396, 677

Heiter, U., Kupka, F., Van't Veer-Menneret, C., Barban, C., Weiss, W. W., Goupil, M.-J., Schmidt, W., Katz, D. \& Garrido, R. 2002, A\&A 392, 619 